\title{
La Psicología Mexicana en el contexto de Iberoamérica
}

\section{The context of Mexican Psychology in Ibero-America}

Fecha de recepción: 04/11/2014

Fecha de aceptación: 15/06/2015

\author{
Rosario Valdés Caraveo \\ Alejandra Tamargo Rivero \\ Escuela Libre de Psicología- Universidad de Ciencias \\ del Comportamiento. Chihuahua, México
}

\section{resumen/alsstract:}

Aunque compartimos un mismo lenguaje y problemas sociales similares el desarrollo de la psicología en los países de Iberoamérica, ha sido un tanto desigual, en algunos países están más desarrollados los gremios de psicología, que en México. Como se dará cuenta en el presente artículo, ostentamos más en la acreditación de programas de psicología. En México el desarrollo de nuestra profesión ha sido con diferentes tendencias y modalidades formativas, asimismo la masificación de nuestra disciplina a partir de la década de los setenta ha hecho que el proceso de calidad de algunos de los programas de psicología se presente en bajos porcentajes, y la organización gremial en cuanto a la certificación de psicólogos ha sido lenta. El presente trabajo es de carácter compilatorio y cita distintos autores que se han preocupado por el análisis de la psicología en México.

Although we share the same language and social problems like the development of psychology in Latin American countries, it has been somewhat uneven, in some Latin American countries the unions of psychology, are more developed than in Mexico. As you will notice in this article, we have more development on the accreditation of programs in psychology. In Mexico, the development of our profession has been with different trends and training modalities , also the rapid grow of our discipline from the seventies has made the process quality of some of the psychology programs are present in low percentages, and the trade organization regarding the certification of psychologists has been slow. This work is compilation character and cites various authors who have been concerned with the analysis of psychology in Mexico.

\section{palabras clave/keywords:}

Acreditación, Certificación profesional, Formación, Tendencias y Psicología en México

Accreditation, Professional certification, Psychology training, Trends and Psychology in Mexico

\section{La psicología en México}

En las primeras décadas del siglo XX hubo una creciente preocupación por la psicología, que se comprueba en los títulos de los libros publicados, las instituciones fundadas y las visitas de científicos extranjeros. Los personajes más importantes en una primera etapa (18961940) son E. Chávez, E. Aragón, E., J. Gómez Robleda y J. Mesa Gutiérrez. De entonces hasta 1958, la psicología se desarrolla principalmente como consecuencia del interés de algunas instituciones estatales, educativas y jurídicas por sus aplicaciones clínicas y psicométricas (Valderrama y Jurado, 1985), como lo muestra el número relativamente grande de tests psicométricos traducidos y adaptados a partir de 1916 (véanse Boder, 1925; Hastings, 
1929 y Colotla, 1982, así como las publicaciones del Departamento de Psicopedagogía e Higiene de la SEP, 1926 y 1930). En las décadas de 1940 y 1950, la psicología es entendida fundamentalmente como una mezcla de psicoanálisis, psiquiatría y psicometría.

La psicología se transforma lenta, pero notoriamente, sobre todo en las décadas de los sesentas y los setentas. En esos años de auge, numerosos psicólogos siguen estudios de posgrado en Estados Unidos y en Europa, cientos de obras de psicología son traducidas en México al español, notables científicos extranjeros enseñan en nuestro país, se organizan regularmente congresos nacionales e internacionales, se publican revistas y se fundan sociedades científicas. Esto sucede, por regla general, en colaboración con psicólogos, universidades y centros de investigación estadounidenses. De modo que la psicología mexicana de este periodo se desarrolla bajo un influjo considerable de la estadounidense. Los autores europeos, especialmente los de Europa oriental, representan una influencia reducida que se abre paso lentamente, sobre todo en los ochentas.

En las décadas de 1940 y 1950, la Psicología es entendida fundamentalmente como una mezcla de psicoanálisis, psiquiatría y psicometría. El periodo que se inicia en 1959 y dura por lo menos hasta 1990 se caracteriza por un enorme auge de la Psicología en muchos sentidos. Tan solo de 1960 a 1987, el número de Escuelas y Departamentos de Psicología pasa de cuatro a 66 y el de Estudiantes de 1500 a 25 000, por lo que hace a los campos de investigación, si en 1960 no había ninguno sistemático, en 1989 se cubre una gama muy amplia.

Autores como Galindo (1959 - 1990). Mencionan que coexisten diferentes escuelas de psicología y psicoanálisis; que a principios de los 70's, la investigación era rara en México y la publicación de resultados más rara aún. El volumen de la investigación y por consiguiente de las publicaciones crece constantemente, sobre todo entre 1975 y 1990, con excepción de un periodo difícil entre 1982 y 1987, que corresponde a los años de la crisis económica de México. No obstante, desde el punto de vista de este autor, a pesar del gran número de enfoques que han existido en México en 30 años, es evidente la existencia de cinco grandes Escuelas en la Psicología: Conductismo, Psicología Transcultural, Psicología Cognoscitiva de orientación norteamericana, Psicología Social de orientación norteamericana y Enfoque Psiquiátrico-psicométrico.

La gran preocupación histórica de los Psicólogos en México ha sido la creación de una Psicología propia, científica y, en los últimos años, socialmente comprometida. Y especialmente se consideran diversos aspectos, entre los que destacan al menos los siguientes:

* El desarrollo de la Psicología como un estudio científico de una parte de la naturaleza. Su objeto de estudio y los métodos de su investigación.

* La evolución de la enseñanza de la Psicología en los diversos grados académicos (Licenciatura, Maestría, Doctorado). Las líneas de investigación y los medios de su difusión (Congresos, Revistas).

* La demanda social de los profesionales de la Psicología, las condiciones de trabajo, los recursos legales, materiales, humanos y económicos, que le afectan. 
* La generación y vida de organizaciones y federaciones de psicólogos, sus objetivos y los medios para alcanzarlos.

La primera sociedad relacionada con la Psicología fue "Sociedad Mexicana de Psicoanálisis" fue fundada en 1956, a la fecha no se documentan registros. Posteriormente en los setentas se constituyen asociaciones de psicólogos con propósitos específicos o paradigmáticas, así surge el Consejo Nacional para la Enseñanza e Investigación de la Psicología (CNEIP) en 1971 y la Sociedad Mexicana de Análisis de la Conducta en 1976, de la misma manera se inicia lentamente la constitución de sociedades estatales o regionales.

Fue hasta finales de la década de los 80's cuando se constituye el Primer Colegio Nacional de Psicólogos (Galindo, 2004), en esta década también se conforman la Sociedad Mexicana de Psicología Social y la Asociación Mexicana de Psicología Social. En los últimos diez años, han surgido una gran cantidad de nuevas organizaciones, en muchas de ellas resulta sin embargo confuso su verdadero objetivo: profesional o comercial.

Con el trabajo del Dr. Rogelio Díaz Guerrero y otros distinguidos psicólogos se forma la Sociedad Interamericana de Psicología en 1951 y está se incorpora como miembro del comité ejecutivo de la Unión Internacional de Psicología Científica (IUPsyS).

La más reciente integración de psicólogos a una organización internacional se realizó cuando el Colegio Nacional de Psicólogos, organizaciones regionales de psicólogos de México, se integran en una organización sudamericana llamada la Unión Latinoamericana de Entidades de Psicología (ULAPSI) Y en el año 2002 el Consejo Nacional para la Enseñanza e Investigación de la Psicología (CNEIP), órgano representativo de todas las Instituciones de Psicología y Asociaciones de Psicología en México funda en colaboración con otras instituciones, la Federación Iberoamericana de Asociaciones de Psicólogos (FIAP).

La importancia de la organización gremial es muy importante en México, sobre todo por el apoyo que las organizaciones existentes han dado a la academia y a la investigación, mediante la realización de congresos y la publicación de revistas científicas (Alcaraz y Bouzas, 1998). Sin embargo, también se reconoce que los colegios no tienen un impacto real en la sociedad mexicana. A partir de 1937, el número de instituciones que ofrecían la carrera de Psicología en el país fue creciendo lentamente hasta 1960, cuando se multiplicaron las instituciones y los planes de estudio que ofrecían esa carrera. En los últimos años, el aumento del número de programas ha sido sorprendente, tal como se puede apreciar en la Tabla 1.

La psicología se configuraba como el estudio plural de los complejos factores que conforman al ser humano. En congruencia con esta visión, se conceptuó al psicólogo como "un profesionista universitario cuyo objeto de estudio es el comportamiento humano individual y social, que a partir de la investigación de los procesos cognoscitivos-afectivos y de su interacción con el entorno, es un promotor del desarrollo humano, consciente de sus responsabilidades éticas para consigo mismo y la sociedad, siendo su función genérica la de intervenir como experto del comportamiento en la promoción del cambio individual y social desde una perspectiva interdisciplinaria (Urbina, 1992). 
Tabla 1.- Crecimiento de escuelas y facultades de Psicología.

\begin{tabular}{|lll|}
\hline Institución formadora & Año de inicio & Número de planes \\
\hline UNAM & 1937 & 1 \\
\hline Iberoamericana & 1950 & 2 \\
\hline D.F. e interior & 1960 & 11 \\
\hline D.F. e interior & 1970 & 54 \\
\hline D.F. e interior & 1990 & 82 \\
\hline D.F. e interior & 1995 & 113 \\
\hline D.F. e interior & 2000 & 183 \\
\hline D.F. e interior & 2005 & 290 \\
\hline
\end{tabular}

Fuente: ANUIES (1966, 1995, 2000, 2006).

Tabla 2.- Etapas en la formación del psicólogo y la constitución de su identidad profesional

\begin{tabular}{|c|c|c|}
\hline Inicio & Tendencia formativa & $\begin{array}{l}\text { Fases de desarrollo de la identi- } \\
\text { dad profesional }\end{array}$ \\
\hline 1937 & $\begin{array}{l}\text { Dependencia de la filosofía y el modelo médico. Claustro } \\
\text { heterogéneo e indefinición. }\end{array}$ & $\begin{array}{l}\text { Identidad confusa } \\
\text { (indiferenciación). }\end{array}$ \\
\hline 1945 & Oscila entre el psicoanálisis y el modelo médico. & Identificaciones contingentes. \\
\hline 1949 & $\begin{array}{l}\text { Tendencia a la medición y la investigación cuantitativa. In- } \\
\text { corporación de estadística, psicometría y técnicas de investi- } \\
\text { gación. }\end{array}$ & $\begin{array}{l}\text { Diferenciación y construcción } \\
\text { de referentes propios. }\end{array}$ \\
\hline 1952 & $\begin{array}{l}\text { Predominio de la formación clínica. Integración de nuevas } \\
\text { orientaciones derivadas de las originales. }\end{array}$ & $\begin{array}{l}\text { Identificación parcial de sus } \\
\text { componentes. }\end{array}$ \\
\hline 1960 & $\begin{array}{l}\text { Tendencia a la psicología experimental y la orientación con- } \\
\text { ductista. }\end{array}$ & $\begin{array}{l}\text { Representación cognitiva } \\
\text { parcial. }\end{array}$ \\
\hline 1970 & Oscilación entre conductismo y psicoanálisis. & $\begin{array}{l}\text { Ambivalencia en representa- } \\
\text { ciones. }\end{array}$ \\
\hline 1977 & $\begin{array}{l}\text { Confluencia de orientaciones diversas, tendencia a la forma- } \\
\text { ción centrada en la disciplina. }\end{array}$ & $\begin{array}{l}\text { Representación cognoscitiva } \\
\text { global. }\end{array}$ \\
\hline 1990 & $\begin{array}{l}\text { Tendencia a incorporar todas las orientaciones sin campos } \\
\text { de especialización. Predomina una orientación teórica con } \\
\text { inclusión de prácticas. }\end{array}$ & $\begin{array}{l}\text { Representación cognoscitiva y } \\
\text { profesional. }\end{array}$ \\
\hline 2000 & $\begin{array}{l}\text { Tendencia a la formación centrada en las prácticas profesio- } \\
\text { nales. }\end{array}$ & Representación profesional. \\
\hline
\end{tabular}

La trayectoria descrita parece confirmar dos supuestos: el primero es que la tendencia en la formación -fuertemente influida por la construcción de la disciplina- está imbricada en la constitución de la identidad profesional, por lo que ambos procesos se influyen mutuamente. El segundo supuesto es que la constitución de la identidad profesional es análoga 
al proceso de constitución de la identidad personal. Al final se logra la integración de las nuevas construcciones y tendencias en una representación global que hace posible proyectarse en la realización a través del desarrollo personal. La constitución de la identidad de la psicología como disciplina y como profesión muestra un proceso semejante (Zanatta y Yuren. 2012).

Los planes de estudio de las escuelas y facultades del interior del país siguieron un proceso semejante al que presentó la UNAM. En general, iniciaron con cátedras en las facultades de Filosofía correspondientes y en el nivel medio superior. Posteriormente, se creó la carrera de Psicología, en algunos casos dependiente de las facultades de Humanidades o Filosofía, y en otros de las de Medicina. Pero en todos ellos el tipo y contenido de materias se vio influido por la composición de la planta docente: médicos, antropólogos, sociólogos y filósofos, los que predominaban respecto a los psicólogos. Al igual que en la UNAM, la hegemonía de un enfoque teórico particular respondió a las relaciones de poder, y la manera en que se resolvieron las tensiones se vio reflejada en los diseños curriculares y los contenidos programáticos.(Harrsch M.A.1994)

El currículo se determinó con base en criterios propios de la disciplina y de lo que los académicos consideraron que el estudiante debiera saber. El plan de estudios incluía de ocho o diez semestres, y por lo general su estructura se conformaba mediante un tronco común y uno especializado, este último en los dos últimos semestres para profundizar en una determinada área o campo profesional (Zanatta y Yuren. 2012). En los años setenta hubo un aumento acelerado del número de escuelas de Psicología en el país: eran once en total en 1970, y para1977 ya eran 54, lo que significó un incremento aproximado de $600 \%$ de la población estudiantil en el país. Lo mismo hubo planes con formación general o con tronco básico y áreas de semiespecialización, que formación general sin tales áreas, o planes de estudio que especializan a lo largo de toda la carrera en una sola área o campo profesional. También se observó esa diversidad respecto a la orientación teórica predominante en el contenido y tipo de materias.

A finales de los años setenta, el Consejo Nacional para la Enseñanza e Investigación de la Psicología (CNEIP) (1977) señaló que era "preocupante la heterogeneidad observada en los currículos de la licenciatura en Psicología". Para el CNEIP, organismo al cual pertenecían y pertenecen la mayoría de las escuelas y facultades acreditadas de psicología del país, la heterogeneidad se debía al crecimiento desproporcionado de escuelas y a la juventud de la profesión y de su enseñanza en México. Para superar esa situación recomendó estructurar un plan de formación de profesores e investigadores en el área de la psicología; definir un perfil profesional del psicólogo en el país; desarrollar modelos curriculares homogéneos que establecieran objetivos generales comunes de formación profesional, elaborados con base en el perfil establecido para el psicólogo mexicano; establecer políticas que regularan la relación ingreso-egreso de estudiantes en las escuelas de Psicología, y detener el incremento de las escuelas y facultades. Tres años después, el CNEIP realizó un análisis de los currículos de la licenciatura en Psicología de la República Mexicana, reportado por Alcaraz (1979). Dicho análisis señalaba lo siguiente: 1) la mayoría de los planes de estudio no contenían objetivos profesionales ni mecanismos claros para evaluarlos; 2)

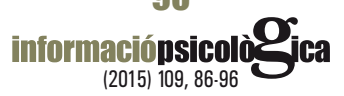


los currículos se basaban en un marco teórico convencional y ofrecían una vasta información al alumno, pero sin tener como meta alcanzar objetivos profesionales precisos; 3 ) las cartas descriptivas abundaban en verbalismos, sin especificar actividades a desarrollar por profesores y alumnos; 4) se privilegiaban los aspectos teóricos en detrimento de la práctica, además de que la formación teórica carecía de un eje rector y que las pocas prácticas que se promovían no eran congruentes con el ejercicio profesional de los egresados; 5) se observaba en los planes de estudio una falta de secuencia lógica entre los contenidos y de congruencia en la seriación vertical, así como una falta de integración vertical y horizontal; 6) existía tal heterogeneidad en las materias que se requería una extensa planta de docentes, lo que dificultaba la comunicación de las academias y la instrumentación de programas de formación docente, y 7) el servicio social no estaba integrado al conjunto de actividades requeridas para asegurar la formación profesional.

Un año después, López, Parra y Guadarrama (1980), al analizar 54 planes de estudio de escuelas de Psicología en universidades mexicanas, reportaron entre sus principales hallazgos lo siguiente: a) los planes de estudio se diseñaron basados en el criterio de los académicos respecto de lo que consideraron importante desde la visión de la disciplina, pero sin llevar a cabo una detección de necesidades y demandas sociales de la profesión del psicólogo; b) $90 \%$ de las escuelas tenía un sistema académico tradicional: organización por áreas y enseñanza tradicional; c) en la mayor parte de las escuelas (58\%), el plan de estudios contenía un tronco básico y áreas de semiespecialización, y d) las escuelas públicas atendían a $74 \%$ de la población estudiantil, en tanto que las privadas tan sólo atendían a $26 \%$.

La búsqueda de unificación y el temor a la diversidad parecen haber tenido algún efecto. A finales de ese decenio, Rivera y Urbina (1989) y Urbina (1992) señalaban que la diversidad de los planes de estudio de la licenciatura en Psicología era aparente, pues un análisis más profundo mostraba que el contenido de muchos de ellos era bastante similar y solamente diferían en la denominación de las materias.

En las últimas dos décadas del siglo XX predominó la tendencia a formar psicólogos generales, lo que tuvo como efecto la eliminación de la especialización temprana. En la mayoría de los programas que tomaron este rumbo se privilegió lo teórico sobre lo práctico, con el resultado de que los egresados no contaban con el "saber hacer" suficiente para resolver problemas en su área de competencia profesional (Castañeda, 1995).

\section{Formación de psicólogos profesionales}

\section{Panorama actual de los programas de psicología}

Las tendencias curriculares de las instituciones de educación superior reflejan que el tipo de dispositivo que se pone en marcha y el diseño curricular no son ajenos a las transformaciones de las condiciones socioeconómicas y culturales del contexto, y que están fuertemente imbrica- dos con los alcances de las profesiones, el desarrollo de los campos ocupacionales y la necesidad de una transformación de la pedagogía universitaria, que está incorporando cada vez más las tecnologías de la información y la comunicación a las prácticas docentes (Zanatta y Yuren. 2012).

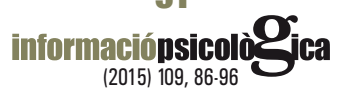


Las transformaciones en la formación profesional también han obedecido a que las universidades públicas han tenido que responder a una demanda creciente de aspirantes a ingresar a los distintos programas de formación. Durante las décadas de los sesenta, setenta y comienzo de los ochenta se reportó un incremento anual de la matrícula de entre 20 y $30 \%$, aproximadamente $230 \%$ cada decenio (Rangel, 1983). Tal situación dio lugar a una masificación de la educación que repercutió en la vida académica y en la calidad de la formación de los estudiantes, toda vez que para atender esa gran cantidad de estudiantes se improvisaron docentes que no tenían el conocimiento de la disciplina ni la capacidad pedagógica requerida. Así, la masificación contribuyó a que el psicólogo en formación pasara la mayor parte del tiempo en el aula y que predominara el dictado en clase como estrategia docente, y condujo también a que se incrementaran los índices de deserción, rezago y reprobación, y al consecuente decremento en el índice de eficiencia terminal. Por otra parte, se presentó el problema de la disparidad entre el número de egresados de nivel superior y la capacidad de absorción del mercado de trabajo (Rangel, 1983). Lo anterior, aunado a la crisis económica de la década de los ochenta y a las políticas internacionales de la Organización de Cooperación y Desarrollo Económico (OCDE), sentó las bases para que la mayor parte de las universidades públicas establecieran como una de sus políticas el frenar el aumento de su matrícula con el argumento de que aceptarían tan solo a la población que podían atender. La selección se efectuó bajo el criterio de aceptar a los estudiantes con mayores posibilidades de éxito, lo que trajo como consecuencia la proliferación de escuelas particulares, en muchos casos de dudosa calidad. De esta forma, se vieron limitadas las oportunidades de acceso a la universidad para los grupos más desprotegidos y se generó una desigualdad que, lejos de resolverse, se va agudizando cada vez más (Zanatta y Yuren. 2012). La necesidad de generar diseños curriculares y metodologías de enseñanza basados en la investigación se planteó desde la década de los setenta. Desde ese momento se puso de manifiesto la escasez de investigadores en el campo curricular y la resistencia de las comunidades universitarias hacia las innovaciones; dichas innovaciones operaron como un factor más que condicionó la formación del psicólogo en las últimas tres décadas del siglo pasado. Aunque nos hemos referido a ellas al mostrar los cambios en ese proceso, a manera de recapitulación se presenta en seguida un apretado resumen de las principales innovaciones recomendadas y de la suerte que corrieron.

En la década de los noventa se diseñaron programas curriculares en tres o cuatro niveles de formación, se reforzó el sistema de créditos en el marco del currículo flexible, se estructuró el plan a través de ejes transversales y longitudinales, y se incluyeron créditos optativos que hacían posible la actualización constante del currículo, la formación interdisciplinaria y un diseño centrado en el estudiante. Asimismo, se promovió la movilidad estudiantil y el intercambio de profesores e investigadores. En casos aislados se diseñaron los currícula con un enfoque centrado en competencias en distintas vertientes: con énfasis en las tareas o la disciplina, o con un enfoque holístico. Algunas instituciones desarrollaron nuevas formas de organización académico-administrativa (departamental o matricial), en lugar de la organización original de escuelas y facultades (ANUIES, 1997). A finales de la década de los noventa y comienzos de la actual se establecieron nuevas directrices para la innovación

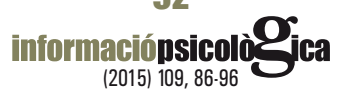




\section{dossier: Psicología lberoamericana - ||}

Rosario Valdé Caraveo, Alejandra Tamargo Rivero

curricular, fundadas en los planteamientos de la conferencia de la UNESCO, celebrada en París en 1998, así como de la ANUIES (2000). Se demandaba una formación más pertinente, sistematizada y polivalente; se enfatizó la necesidad de procurar la formación integral del estudiante considerando la formación en valores y el desarrollo personal que coadyuvaran a elevar la calidad de vida del estudiante, y se recomendó un sistema tutorial como acción estratégica para el logro de todo ello.

\section{Calidad de los programas de psicología (CNEIP)}

El Consejo Nacional para la Enseñanza e Investigación en Psicología (CNEIP), es un organismo asesor en lo académico de las Carreras de Psicología de México sin fines de lucro, únicamente dedicado primordialmente entre algunos de sus objetivos a:

- Orientar la enseñanza e investigación de la psicología a la solución de los problemas sociales que plantea la realidad nacional en lo que a esta disciplina respecta.

- Contribuir en el campo de la Psicología, a la implementación de las políticas y normas contempladas en la Ley Federal de Educación de la República Mexicana.

- Impulsar la enseñanza, investigación, difusión y el ejercicio profesional de la psicología científica en las instituciones de enseñanza y/o de investigación de la República Mexicana.

- Otorgar la acreditación a los programas educativos que satisfagan los estándares de calidad en la formación de los profesionales de la psicología, en los niveles de licenciatura y posgrado.

El Consejo Nacional para la Enseñanza e Investigación en Psicología está reconocido por COPAES como un organismo acreditador de Programas Educativos en Psicología El Consejo Nacional para La Acreditación de la Educación Superior, A. C. (COPAES) "es la instancia capacitada y reconocida por el Gobierno Federal a través de la Secretaría de Educación Pública para conferir reconocimiento formal a favor de organizaciones cuyo fin sea acreditar programas académicos de educación superior que ofrezcan instituciones públicas y particulares, previa valoración de su capacidad organizativa, técnica y operativa, de sus marcos de evaluación para la acreditación de programas académicos, de la administración de sus procedimientos y de la imparcialidad del mismo" (COPAES, 2011). El COPAES es una asociación civil de carácter no gubernamental cuya misión es contribuir al aseguramiento de la calidad de los programas académicos que se ofrecen en las instituciones públicas y particulares de México, mediante el reconocimiento formal de las organizaciones de acreditación que demuestren la idoneidad, calidad y confiabilidad de sus procesos y resultados, y que desarrollen sus funciones y procesos con base en los Lineamientos y en el Marco General para los Procesos de Acreditación de Programas Académicos de Nivel Superior, establecidos por el mismo Consejo. (COPAES, 2011). A la fecha existen 93 programas de Psicología Mexicanos acreditados de los 948 que están registrados en el padrón. (CA-CNEIP, 2014) 


\section{Acreditación por el Consejo Nacional de Enseñanza elnvestigacion en Psicologia (CNEIP)}

El Comité de Acreditación del Consejo Nacional para la Enseñanza e Investigación en Psicología (CADCNEIP) es el órgano del CNEIP, responsable de realizar la evaluación y emitir el dictamen de acreditación de los programas educativos de Psicología, en los niveles de Técnico Superior Universitario, Licenciatura y Posgrado y con autonomía para realizar su función.

Este recibió el refrendo de comité acreditador autorizado en el año 2014, por el Consejo para la Acreditación de la Educación Superior, A. C. (COPAES), que es la instancia del Gobierno Federal a través de la Secretaria de Educación Publica (SEP) para conferir el reconocimiento formal a organizaciones cuyo fin sea acreditar programas académicos de educación superior que ofrezcan instituciones públicas y particulares.

\section{El proceso de Acreditación de CA-CNEIP}

La acreditación de un programa de Psicología requiere de un proceso voluntario, objetivo, justo, transparente, externo, ético, responsable, confiable, colegiado, integral y temporal, y se realiza siempre con base en el reconocimiento de la diversidad institucional existente en la educación superior.

\section{Etapas}

El proceso de Acreditación se conforma por seis etapas que se vigilan cercanamente.

1. Autoevaluación

2. Solicitud de Acreditación

3. Pago de evaluación

4. Evaluación del CA $\square$ CNEIP

5. Revisión y confirmación por CA $\square$ CNEIP de que la documentación presentada avala el cumplimiento de los prerrequisitos e imprescindibles por la Institución.

6. Evaluación in situ por los evaluadores designados por el CA $\square$ CNEIP

- Dictamen.

- Entrega del Diploma, dictamen y recomendaciones

Las categorías que están incluidas en el marco de referencia 2014 son:

1. Personal académico

2. Estudiantes

3. Plan de estudios

4. Evaluación del aprendizaje

5. Formación integral

6. Servicios de apoyo para el aprendizaje

7. Vinculación - extensión 
8. Investigación

9. Infraestructura y equipamiento

10. Gestión administrativa y financiera

- Anexos

- Seguimiento de recomendaciones

\section{La psicología organizada en México}

El registro de profesionales de la psicología se lleva a cabo desde el año de 1974 y a la fecha existen registros solo por la licencia otorgada por la Dirección General de Profesiones (DGP), la llamada "Cedula Profesional”. Existe actualmente el Colegio Nacional de Psicólogos desde hace algunas décadas pero aún no ha regulado la profesión. Recientemente la SEP convoca a grupos disciplinarios para realizar la "Certificación de los psicólogos en México" a raíz de esta se creó el Colegio Mexicano de Psicología (COMEPSI) auspiciado por la Sociedad Mexicana de Psicología quienes han emprendido la tarea de iniciar la Certificación de profesionales manera formal. Se ha reportado también que el Colegio Nacional de Psicólogos (CONAPSI) está trabajando en colaboración con CNEIP en esta ardua tarea. Solo existe actualmente el registro de la Dirección General de Profesiones en México de Cedulas Profesionales expedidas a Licenciados en Psicología, sin considerar la actualización de los mismos. La importancia de la organización gremial recientemente ha sido reconocida, sobre todo por el apoyo que las organizaciones existentes han dado a la academia y a la investigación, mediante la realización de congresos y la publicación de revistas científicas (Alcaraz y Bouzas, 1998). Sin embargo, también ha sido reconocido el hecho de que la psicología mexicana carece de una tradición corporativa, que los colegios no tienen un impacto real en la sociedad mexicana comparado con el trabajo que han realizado organizaciones gremiales de otras disciplinas, que muchas organizaciones tienen una corta vida y que las que se han mantenido plenamente consolidadas no cuentan con una base de miembros estable y participativa. (Vargas y Aguilar, 2007).

\section{Conclusión}

A lo largo de este documento compilatorio nos hemos podido percatar, que si bien la psicología en México ha sido influenciada por distintas vertientes teóricas y prácticas, así como diversas escuelas y universidades. El tiempo ha permitido hasta cierto punto un consenso en cuanto al protocolo para la educación y la ética dentro de la psicología. Así mismo no es difícil darse cuenta que existe solo un organismo que acredita a las instituciones de la psicología, y varios que pretenden certificar a los profesionales de la misma por lo cual sería conveniente trabajar en un único organismo que nos permita regular y hacer más eficientes los procesos dentro de la psicología. Es importante mencionar que la psicología es bastante rica en perspectivas teóricas ya que tenemos una gran influencia de distintos países: por nombrar algunos EUA, países latino americanos y algunos europeos, por lo cual el potencial de crecimiento para nuestra ciencia es muy bueno tanto en áreas de la psicología clínica, salud, social y experimental. En un futuro próximo sería conveniente que la psicología

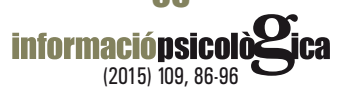


en México trabaje en la legislación de artículos que permitan la regulación y un adecuado manejo de la formación de recursos humanos en psicología, así como de la regulación de la práctica profesional.

\section{Referencias}

Alcaraz,V. (1979). Resultados de los talleres de Cocoyocy de San Miguel Regla. Enseñanza e Investigación de la Psicología, 5 (1), $678-687$.

Alcaraz, V. y Bouzas, A. (Eds.) (1998). Las Aportaciones Mexicanas a la Psicología: La Perspectiva de la Investigación. UNAM, U de G. México, D. F.

Asociación Nacional de Universidades e Instituciones de Educación Superior (1966). Anuario estadístico. México: ANUIES.

Asociación Nacional de Universidades e Instituciones de Educación Superior (1995). Anuario estadístico. México: ANUIES.

Asociación Nacional de Universidades e Instituciones de Educación Superior (1997). Anuario estadístico. México: ANUIES.

Asociación Nacional de Universidades e Instituciones de Educación Superior (2000).Anuario estadístico. México: ANUIES.

Asociación Nacional de Universidades e Instituciones de Educación Superior (2006). Anuario estadístico. México: ANUIES.

Castañeda, S. (1995). Los problemas de la educación superior y la formación del psicólogo.Perfiles Educativos, 68. Disponible en línea: http://redalyc.uaemex.mx/ pdf/132/13206802.pdf.

Consejo Nacional para la Enseñanza e Investigación de la Psicología en México (1977). Recomendaciones generales para la planeación de la enseñanza de la Psicología en México. Enseñanza e Investigación en Psicología, 3 (2), 21-24.

Consejo Nacional para la Enseñanza e Investigación de la Psicología en México (2000- 2007). Memorias de Asambleas Anuales.

Galindo, E. (2004). Análisis del desarrollo de la psicología en México hasta 1990: Con una biografía en extenso. Psicología para América Latina, (2).Recuperado de: http://psicolatina.org/Dos/analisis_psicol.html

Harrsch, C. (1994). Identidad del psicólogo. México: Alhambra Mexicana.

López, A., Parra, G. y Guadarrama, L. (1980). Análisis curricular de la enseñanza de la Psicología en México. Tesis inédita de Licenciatura. Toluca (México): UAEM.

Rangel, G. (1983). La educación superior en México (2a ed.). México: El Colegio de México (Col. Jornadas, n. 86).

Rivera, R. y Urbina, J. (1989). Estadísticas básicas sobre la formación de psicólogos en México. En J. Urbina (Comp.): El psicólogo: formación, ejercicio profesional y prospectiva. México: UNAM.

Urbina, J. (1992). El psicólogo, formación, ejercicio profesional y prospectiva. México: Universidad Nacional Autónoma de México.

Valderrama, L. y Jurado, C. (1985) La psicología aplicada al estudio y tratamiento de la delincuencia en México $1920-$ 1940. Revista Mexicana de Psicología. 2, 2(4), 176-187.

Vargas, J. y Aguilar, J. (2007). La Psicología en México: Organización, Poder, Control Para qué? (La historia de las asociaciones de psicólogos en México). México. Boletín electrónico de la Asociación Oaxaqueña de Psicología, 3(1), 5-14.

Zanatta, C. y Yuren, C. (2012) La Formación profesional del psicólogo en México: trayecto de la construcción de su identidad disciplinar. Enseñanza e Investigación en Psicología, 17(1); 151-170 